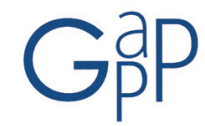

GESTIÓN Y ANÁLISIS DE POLÍTICAS PÚBLICAS, Nueva Época, nº 13 enero-junio 2015 ISSN: 1989-8991

DOI: http://dx.doi.org/10.24965/10.24965/gapp.voi13.10233

\title{
Estrategias participativas para el desarrollo integral de la producción forraje-leche en la región de Tulancingo (México)
}

\author{
Yolanda Sánchez Torres \\ Universidad Autónoma del Estado de Hidalgo, México \\ yolasato08@hotmail.com
}

Aníbal Terrones Cordero

Universidad Autónoma del Estado de Hidalgo, México aterrones68@hotmail.com

\section{Resumen}

El desarrollo rural integral requiere del involucramiento de los diferentes agentes que interactúan dentro de un territorio, puesto que ello posibilita el aprovechamiento de su potencialidad a favor de un mayor desarrollo económico y social. En este sentido la planeación participativa se convierte en una herramienta importante para el alcance de esta meta. El presente estudio muestra como los procesos participativos para la producción de forraje-leche, en la región de Tulancingo, permitió generar estrategias que atendieran la problemática central de la falta de eslabonamiento productivo de la actividad láctea. Para ello se determinaron tres ejes estratégicos en común: aumento en la calidad y rendimiento del producto, recuperación del margen de utilidad, y focalización de los programas gubernamentales. Además de la provisión adecuada de infraestructura carretera y equipamiento, fomento al trabajo colaborativo y capacitación de calidad. Esta metodología posibilitó el diseño de estrategias de eslabonamiento productivo de abajo hacia arriban, en el corto y largo plazo; pero sobre todo, permitió el reconocimiento de la responsabilidad de gestión que asumen los diferentes actores para un desarrollo coordinado e incluyente.

\section{Palabras clave}

Forraje-leche, eslabones productivos, planeación participativa, políticas públicas, desarrollo regional.

\section{Participative strategies for the integral development forage-milk of the production in the region of Tulancingo (Mexico)}

Abstract

The integral rural development requires of the involvement of the different agents who interact within a territory, because it makes possible the advantage of its potentiality in favor of a greater economic and social development. In this sense the participative planning it becomes an important tool for the reach of this goal. The present study shows how the participating processes for the forage-milk production, in Tulancingo's area, have allowed generating strategies to consider the central problematic of productive linking inexistence of the milky sector. Thus there were three strategic axes in common: increased quality and yield, the profit margin recovery, and focalization of government programs. Besides providing adequate road infrastructure and equipment, foment the collaborative work and quality training. This methodology made possible the design of strategies of productive chains from down to the top, in a short and long term, but mainly, it allowed the recognition of the management responsibility that assumes the different actors for a coordinated and inclusive development.

Key words

Forage-milk, productive linking, participative planning, public policies, regional development. 


\section{INTRODUCCIÓN}

El desarrollo económico nacional se fundamenta en las estrategias locales, los gobiernos municipales democráticos constituyen un sistema fundamental para alcanzar el desarrollo regional y, con ello, nacional.

\section{Referente Teórico}

Pardo (1994), Sánchez et al. (2003) y Silva (2003) sostienen que el crecimiento y desarrollo económico de una sociedad implica necesariamente procesos continuos de cambios estructurales en el ámbito productivo, político y social de las diferentes regiones de un determinado país. Entendiendo a la región como un espacio geográfico donde se realizan actividades productivas con presencia de actores sociales y políticos que promueven el crecimiento económico (Ramírez, 2004).

Vázquez y Conturruero (1994) y Blakely y Green, (2010) mencionan que los procesos de crecimiento y desarrollo económico de una sociedad se deben construir desde abajo, es decir, a partir de un diagnóstico participativo sobre las necesidades y potencialidades de las comunidades, en cuanto a los recursos humanos, naturales y materiales que prevalecen en una determinada región. Giménez (1994), Terrones y Sánchez (2010) y Terrones (2013), señalan que existe una marcada interdependencia entre la región y los actores sociales, por lo que estos últimos ejercen un rol importante en el desarrollo económico y social de una determinada región, al ayudar a entender a la gente que la habita, sus necesidades, su historia, su problemática y sus posibles soluciones.

La Organización para la Cooperación del Desarrollo Económico (OCDE, 2011), sostiene que los diagnósticos de abajo hacia arriba con visión territorial pueden ayudar a identificar potencialidades sectoriales desaprovechadas a favor de la diversificación económica, la derrama sectorial y dinámica regional. Por ello, deben fomentarse políticas públicas innovadoras, basadas en el territorio y en procesos participativos que se adapten a las características y oportunidades específicas y variadas de las diferentes regiones de un país. En caso contrario, se profundiza la concentración económica y demográfica que involucra por sí misma la diversificación regional, reflejada en grandes disparidades intrarregionales.

La determinación de las causas de los problemas y la identificación de las potencialidades productivas de una comunidad, permiten diseñar estrategias de desarrollo regional y, con ello, mejorar las condiciones de bienestar de la población (Morales, 1998; Geilfus, 2000; Terrones, 2013).

La planeación en el medio rural es eficiente y eficaz siempre y cuando considere las características fundamentales de cada territorio y participen activamente todos los involucrados, tanto en la identificación de necesidades y prioridades comunes; así como en la elaboración, ejecución y seguimiento de alternativas de solución, plasmadas en las políticas, planes, programas y proyectos (Schönhuth, 1994). Cuando estos elementos no son considerados se generan procesos exógenos o verticales "de arriba hacia abajo", que no responden a los intereses ni a la realidad de los productores (Rodríguez et al., 2015).

Ramírez (2004) y Osorio (2009), usando un esquema participativo, plantearon la necesidad de realizar una planeación en consenso de los proyectos con el fin de priorizar actividades, definir fechas, responsables de la ejecución y monitoreo de las diferentes actividades.

Terrones y Sánchez (2010), aplicando la Planeación Participativa en el diseño de estrategias de desarrollo económico y social en Actopan Hidalgo, y Terrones (2013) en el municipio de Acaxochitlán Hidalgo; concluyeron, con relación al fomento pecuario, que es necesario: crear y mejorar la infraestructura en la producción pecuaria; mejoramiento genético (mejores razas); y financiar con recursos suficientes a dicha actividad. Con relación al fomento agrícola, determinaron que se deben de otorgar apoyos institucionales suficientes para desarrollar la agricultura; utilizar variedades de semillas mejoradas; métodos de riego óptimos en la actividad agrícola; y dar asesoría técnica a los productores agrícolas.

El desarrollo agropecuario representa un mecanismo para aumentar los ingresos y reducir la pobreza en las zonas rurales. Esto implica disponibilidad de financiamiento y de tecnologías a pequeños y medianos productores del campo (Banco Mundial, 2005: 34). La actividad agropecuaria en México se caracteriza por su baja productividad, de autoconsumo, uso de tecnologías inadecuadas, sistemas de financiamiento gubernamental ineficientes. Ante esto, es necesario reactivar la producción en el campo mediante la identificación de problemáticas y propuestas de solución desde el punto de vista de los propios productores con el apoyo de las diferentes instituciones encargadas de fomentar el desarrollo del medio rural (Martínez et al., 2014).

La presente investigación se conceptualizó dentro de la cadena productiva de producción de lácteos, entendida como el eslabonamiento entre actividades productivas con diferentes funciones desde la producción primaria, 
la transformación industrial hasta la distribución de productos finales, sin dejar a lado la proveeduría de bienes y servicios (FAO, 2013; Sagarpa, 2013). Para este caso se analizaron únicamente los dos primeros eslabones (forrajeleche-quesos) para la región Tulancingo. El estudio se presentó de esta manera porque la caracterización de la cadena en la región permite ver un eslabonamiento directo entre forraje-leche, pero una separación e incluso enfrentamiento con el eslabón de la industria de la transformación de productos derivados o finales de la leche, especialmente de quesos.

\section{Contexto geográfico y socioeconómico}

La región de Tulancingo se ubica al noroeste del Estado de Hidalgo. La integran los municipios de Tulancingo de Bravo, Santiago Tulantepec, y Cuautepec de Hinojosa. Cuenta con una superficie total de 771.44 km², que representa el 3.71\% de la superficie y el 8.98\% de la población estatal (Inegi, 2010).

Dentro de las principales actividades económicas en la región, destaca la textil, comercial y agropecuaria, y en esta la cadena productiva de lácteos. Según el Sistema de Información Agropecuario (SIAP, 2013), en 2011, el maíz grano ocupó el 38.31\% de la superficie sembrada, seguida por los cultivos forrajeros: avena forrajera en verde (18.9\%), prados y praderas en verde (9.3\%), alfalfa verde (3.2\%), y maíz forrajero en verde (2.7\%). Lo anterior permite reafirmar la importancia que tiene este eslabón en la cadena productiva forraje-leche, puesto que la mayoría del destino de la producción de cultivos forrajeros en la región se utiliza para la alimentación de ganado bovinolechero.

El sector lechero es muy importante en Hidalgo, ocupa el octavo lugar a nivel nacional, con una participación del 4\%. Por su parte, la región Tulancingo en 2011, representó el 6.91 \% de la producción de leche estatal; destacando Tulancingo con una producción de 13 millones 438 mil litros de leche (3.37\% estatal), ocupando el tercer lugar; mientras Santiago y Cuautepec ocuparon el décimo y décimo primer lugar respectivamente (SIAP, 2013).

El primer eslabón de la cadena productiva (forraje-leche-queso) en la región Tulancingo, se caracteriza por una producción empírica, con régimen hídrico de temporal, estructura minifundista (4 hectáreas en promedio), métodos de producción poco mecanizados y baja rentabilidad que se maximiza al destinar su producción al siguiente eslabón de la cadena (leche bovino). Este segundo eslabón se caracteriza como una actividad empírica, de pequeña escala, con bajos estándares de calidad que demeritan el precio de comercialización en el siguiente eslabón de la cadena productiva para la transformación industrial (queso).

Para la región Tulancingo, la problemática del sector forrajero se transfiere y multiplica al siguiente eslabón, puesto que se produce forraje por la utilidad que tiene como alimento para el ganado bovino, el cual provee leche como un bien comercializable. Es aquí donde se define si la actividad es productiva o no, si les provee un mejor nivel de bienestar o simplemente un empleo, si continúan o deciden terminar. Esta disyuntiva se ha visto agravada en las últimas dos décadas, como lo manifiestan los productores de forraje-leche en la región de estudio, las posibilidades no de competir, sino sobrevivir, son cada vez menos, por lo que se requiere diseñar e implementar nuevas estrategias que permitan la reactivación de esta actividad económica y el eslabonamiento y competitividad de esta cadena productiva.

En esta investigación se diseñaron las estrategias de desarrollo en la producción de forraje-leche para dicha región, con el fin de mejorar las condiciones socioeconómicas de los productores en la región Tulancingo Hidalgo, utilizando la Planeación Participativa. Este sistema participativo permitió identificar las potencialidades y áreas de oportunidad de los productores de forraje-leche en la región de estudio, estableciendo además los lineamientos de solución.

\section{METODOLOGÍA Y TALLERES PARTICIPATIVOS}

Olvera (1999) y Osorio (2009), señalan que la participación ciudadana es uno de los elementos que contribuyen a profundizar la democracia, pero sobre todo que posibilita la mejora en las condiciones de vida de la población, ante la ineficiencia en los procesos de planeación y falta de representatividad del sistema de planeación tradicional, que se caracteriza por diseñar, ejecutar y dar seguimiento a lineamientos establecidos de manera unilateral. Por ello, es necesario considerar las diferentes opiniones y decisiones de las personas, líderes, y representantes de organizaciones sociales e instituciones públicas, en el diseño, realización y seguimiento de proyectos específicos a favor de un mayor desarrollo económico regional integral y coordinado.

Los procesos participativos, según Banco Mundial (citado por Uemura, 1999), involucran cuatro etapas de participación: compartir información, consultar, compartir el control de las decisiones y tomar acción. Siendo las dos primeras etapas, prerrequisitos para la participación y las últimas dos la esencia y el resultado de la parti- 
cipación. Estas determinan la tipología y alcance de los procesos participativos, en diferentes enfoques como los presupuestos participativos, la gestión participativa, la gestión por resultados, entre otros. Dentro de estos procesos participativos, Geilfus (2000) considera cuatro tipos de herramientas que deben considerarse según las necesidades y realidades de la comunidad, y de la institución de desarrollo. Estas son: técnicas de dinámica de grupos, visualización, entrevistas y comunicación oral y, observación de campo. La técnica de grupo es fundamental para trabajar con grupos de personas y lograr su participación efectiva. Por su parte, las técnicas de visualización', utilizadas por la planeación participativa, consisten en representaciones gráficas, donde se logra la participación de personas con diferentes grados y tipos de educación, y se facilita la sistematización de conocimientos y en consenso.

La planeación exitosa debe partir del conocimiento pleno de la realidad y del contacto permanente con la población objeto de estudio. Por lo que con la aplicación del método ZOPP (por sus siglas en alemán Ziel-Orientierte Projekt-Planung, en español: Planeación de Proyectos Orientada a Objetivos), o comúnmente denominado Planeación Participativa, se pueden identificar prioridades comunes y diseñar estrategias y acciones adecuadas para impulsar el desarrollo regional integral (Forster y Osterhaus, 1995).

En 1981, la Agencia Alemana de Cooperación Técnica: Team Technologies (GTZ) aplicó el ZOPP en la planeación de proyectos, este enfoque se difundió por todo el mundo. Lo han adoptado, entre otros organismos internacionales, la Organización Internacional del Trabajo (OIT), el Programa de las Naciones Unidas para el Desarrollo (PNUD), la Organización Mundial de la Salud (OMS), la Organización Panamericana de la Salud (OPS), la Organización de las Naciones Unidas para la Agricultura y la Alimentación (FAO), el Banco Interamericano de Desarrollo (BID), y el Banco Mundial (GTZ, 1995).

El gobierno de Nigeria aplicó el método ZOPP en la planificación de la producción agrícola en el norte del país, encontró que dicha actividad se desarrollaba con nula o baja tecnificación y sin acceso al crédito gubernamental, por parte de los campesinos, esto ocasionaba niveles muy bajos de productividad en el campo y, por lo tanto, ingresos insuficientes que no permitían el desarrollo de las familias. De las estrategias de solución, a esta problemática, fue el de proporcionar créditos, insumos y maquinaria agrícola a los productores (Leurs, 1996).

Por su parte, el gobierno de El Salvador utilizó esquemas participativos para determinar y priorizar problemas a nivel de comunidad, desarrollando las estrategias de solución, teniendo como resultado una mayor disponibilidad de servicios públicos básicos y aumento en la credibilidad y respeto hacia las autoridades locales y federales (Rodríguez, 1990).

En el municipio de Bello (Antioquia, Colombia), Rodríguez et al. (2015) generaron una propuesta metodológica para la formulación participativa de un Programa Agropecuario Municipal, con adaptación de la técnica de análisis estructural prospectivo de grupos focales para la implementación de talleres participativos que permitieran determinar las variables clave que requerían acción prioritaria (37 variables agrupadas en seis grupos o factores) con el fin de integrar, de forma activa, a la comunidad en la identificación de la problemática del sector agropecuario y en el planteamiento de estrategias de solución que garantizaran la sostenibilidad de los procesos de desarrollo rural en el Bello, Colombia.

En el caso de México, el método ZOPP lo ha implementado la Comisión Nacional del Agua (Conagua) para la elaboración de planes integrales del uso y manejo de aguas en los diferentes acuíferos del país, y que han servido de base para la elaboración de la ley de aguas nacionales. Gobiernos municipales y estatales lo han empleado para la elaboración de los planes de desarrollo, con la colaboración de instituciones de enseñanza e investigación (Terrones y Sánchez, 2010; Terrones, 2013).

Los instrumentos de análisis del ZOPP son: Árbol de Problemas, Árbol de Objetivos, Análisis de Involucrados, Matriz de Planeación de Proyecto y Planeación Operativa de Proyecto; cuya estructuración implica la realización de talleres participativos, denominados talleres ZOPP.

1 Las técnicas de visualización son: a) matrices, presentadas en cuadros que permiten ordenar y presentar las informaciones e ideas en forma lógica, para fines de cruzar diferentes criterios (matrices de clasificación y de priorización) o de presentar ideas en forma jerárquica (matrices de planificación y otras); b) mapas, representaciones simplificadas de la realidad que tienen aplicaciones en las fases de diagnóstico y análisis, muchas veces sirven de punto de partida para los procesos de desarrollo; c) flujogramas, son un tipo de diagramas que se presenta en forma esquemática, las relaciones entre diferentes elementos (simbolizados por flechas) como relaciones de causa a efecto y secuencia de eventos, y d) diagramas temporales, son representaciones de la presencia/ausencia o de la variación en intensidad de ciertos fenómenos en el tiempo. 


\section{a. Árbol de Problemas}

Osterhaus y Salzer (1995) mencionan que el diseño de proyectos responde a la presencia de un problema de desarrollo, un obstáculo al desarrollo. Muchas veces hay consenso de que la situación actual es insatisfactoria, que se requiere un cambio. Terrones (2013) determinó que el Árbol de Problemas es el diagnóstico de la situación actual, realizado a partir de la identificación del problema central y la visualización y análisis de las causas y efectos que origina dicho problema. Un problema se expresa en términos de la carencia de determinados elementos, como se ilustra en la Figura 1 en cuanto a la falta de organización, programas de gobierno inadecuados, entre otros.

\section{b. Árbol de Objetivos}

A este también suele llamarse Árbol de Decisiones, describe la situación deseada a la que se quiere llegar mediante la solución de los problemas, transformando las relaciones causa-efecto en relaciones medios-fines. El Árbol de Objetivos se forma convirtiendo el problema que está en el nivel más alto del Árbol de Problemas a un objetivo. Luego se sigue trabajando hacia abajo, convirtiendo cada causa en un medio de abordar el problema de desarrollo, formulando cada condición negativa como una condición positiva, es decir, objetivos que son deseados y factibles en la realidad, como se observa en la Figura 2.

\section{c. Análisis de Involucrados}

Al diagnóstico de los principales actores que participan en el proceso de planeación se le llama Análisis de Involucrados. En un proyecto o programa pueden estar involucradas muchas dependencias gubernamentales y no gubernamentales; de orden público o privado; organizaciones de base o de apoyo, de producción o de servicios. Cada una tiene sus propios objetivos e intereses, su propia cultura organizacional, y con frecuencia no es fácil armonizar los diversos intereses. Se analizan todas las personas, grupos y organizaciones involucradas directa e indirectamente con el proyecto, indicando sus funciones, intereses, fortalezas y debilidades. Se examina la realidad social y las relaciones de poder. Los planificadores tendrían que distinguir entre los involucrados activos (líderes) y pasivos (con poca participación o que no asisten pero se presentan sus representantes).

\section{d. Matriz de Planeación de Proyecto}

La Matriz de Planeación de Proyecto (MPP) expresa, de manera integrada, la estrategia de ejecución del proyecto, misma que comprende la definición del Objetivo Superior, Objetivo del Proyecto y las Actividades, así como sus Indicadores Verificables Objetivamente y Supuestos, como se ilustra en el Cuadro 1 y 2.

La solución a la problemática general planteada se especifica en el Objetivo Superior. El Objetivo de Desarrollo concentra la atención de todos los actores involucrados en el proyecto en el proceso de desarrollo de los grupos destinatarios, los proyectos se llevan a cabo para fomentar procesos de cambio que afectan siempre a determinadas personas y organizaciones. La función del Objetivo de Desarrollo es dar una perspectiva conjunta al proceso de cambio que se aspira impulsar. El Objetivo del Proyecto describe el cambio que se desea introducir en la actuación de personas u organizaciones.

Los pasos específicos que conducen hacia la obtención de un resultado son las Actividades. Es necesario tener una lista detallada de las Actividades ya que será la base para la elaboración de la Planeación Operativo del Proyecto. Se coloca cada Actividad requerida para un componente en orden cronológico y se estima el tiempo y recursos requeridos para realizarla.

Los Indicadores Verificables Objetivamente permiten tener una idea sobre el nivel de exigencias y los criterios de éxito del proyecto, están relacionados a los Objetivos, Resultados y Supuestos del proyecto. Los Indicadores pueden referirse a productos físicos (por ejemplo, aumento del rendimiento), o a cambios en la organización (por ejemplo, vinculación interinstitucional). Los Supuestos son situaciones externas que tienen que estar dadas para que un proyecto tenga sentido y sea realista. El riesgo se expresa, convencionalmente, como un Supuesto que debe ocurrir.

\section{e. Planeación Operativa de Proyecto}

La desagregación de las Actividades, determinadas en la MPP, en Sub-actividades se plasma en la Planeación Operativa de Proyecto, esta contiene una asignación de atributos que permiten hacer administrables o monitoreables las Actividades, como Resultados esperados, Fechas de Ejecución, Responsables y los Supuestos.

Con el fin de obtener los Resultados de un proyecto, en ocasiones, es necesario establecer las Sub-actividades, que implica realizar una acción específica dentro de una Actividad, esto permitirá el cumplimiento de lo 
considerado en dicha Actividad. El éxito de la Planeación Participativa radica en llevar a cabo las Sub-actividades de manera adecuada.

Los Resultados son los productos (bienes y servicios) que aportan las organizaciones que ejecutan el proyecto para que puedan producirse los cambios esperados en los receptores de la contribución (Objetivo del Proyecto). Son las obras, estudios, capacitación, padrones, base de datos, etc., que debe producir el proyecto con el presupuesto asignado. Cada componente debe ser necesario para lograr el propósito y debe ser razonable suponer que si todos los componentes son producidos de la manera planeada se cumplirá el fin.

Los Medios de Verificación describen las fuentes de información necesarias para la recopilación de los datos que permiten el cálculo de los Indicadores. Estos constituyen la base del sistema de monitoreo del proyecto. El sistema de monitoreo y evaluación describe los niveles, personas, eventos, procedimientos, documentos y datos que deben ser usados para realizar el seguimiento de la ejecución del proyecto.

La Planeación Operativa de Proyecto especifica la contribución que deben prestar los diversos actores, y pone en claro las respectivas responsabilidades. También establece las Fechas de Ejecución de las diferentes Actividades con el fin de cumplir en tiempo y forma los Objetivos de los proyectos considerados.

\section{f. Talleres ZOPP}

El instrumento de aplicación de los talleres ZOPP logra un consenso en la forma de considerar la situación, se pone de manifiesto las diferencias entre distintos intereses y puntos de vista. Además, los talleres han demostrado ser eficaces para aclarar una situación de necesidad y estrategias de solución directamente con los afectados. El tiempo necesario para la realización de los talleres ZOPP depende de, entre otros factores, la problemática a tratar, la dinámica de trabajo, la logística de los talleres (lugar de realización de los talleres, disponibilidad de los participantes), cobertura de la planeación y apoyos económicos. En general, el tiempo promedio para la realización de los talleres de Planeación Participativa es de 30 a 48 horas (GTZ, 1995; Terrones, 2013).

En este caso la Planeación Participativa se utilizó para el diagnóstico de la problemática y diseño de estrategias de desarrollo de la producción de forrajes-leche en la región Tulancingo. Para ello, se realizaron un total de seis talleres ZOPP, en donde participaron diversos involucrados, que se mencionan en Resultados, que permitieron plasmar la problemática de esta actividad productiva, así como las estrategias para un desarrollo integral en la región de estudio. Cabe señalar que el número de talleres estuvo determinado por la misma dinámica del sector y su respuesta de convocatoria; sin embargo, fueron suficientes para alcanzar el objetivo del presente estudio, permitiendo la interacción de los involucrados y la validación de la información dentro de los talleres para la construcción de los diferentes instrumentos de análisis como lo exige la metodología.

\section{ANÁLISIS DE RESULTADOS}

Con el fin de determinar la problemática y, con ello, diseñar lineamientos de solución de los productores de forrajes y leche de la región Tulancingo, se realizaron un total de seis talleres ZOPP los días 10, 12, 18, 24, 26 y 30 de abril de 2013 con una duración total de 36 horas, fungiendo como moderadores y responsables de dichos talleres los autores de la presente investigación. El tiempo y número de ellos, fueron en apego a la metodología y sobre todo acorde a la naturaleza de los requerimientos del sector de estudio para el alcance del objetivo de la presente investigación.

A los talleres asistieron productores de forraje-leche, representantes de organizaciones de productores de forraje-leche, autoridades municipales (Desarrollo Agropecuario Municipal, Desarrollo Económico y Medio Ambiente), Delegados Municipales y Comisariados ejidales. Así también, asistieron representantes de la Universidad Politécnica de Tulancingo; Universidad Autónoma del Estado de Hidalgo; Secretaría de Desarrollo Económico del Estado de Hidalgo (Sedeco-Hidalgo); Representantes y Administradores de Centros de Acopio de Leche; Fideicomisos Instituidos en Relación con la Agricultura (FIRA); Cámara Nacional de la Industria y la Transformación (Canacintra), Consejo Consultivo Ciudadano-Tulancingo, Tulancingo no te Quedes Mirando, entre otros.

La participación de los diferentes involucrados en los talleres corrobora la presencia de agentes de carácter público y privado, de tipo empresarial, gubernamental, académico y como ciudadano. De tal forma que su interacción permitió una retroalimentación importante sobre la problemática y estrategias de solución en esta actividad productiva, generando sinergias interesantes al interior de los talleres.

Dentro de las limitantes de los procesos participativos, siempre va estar latente la concurrencia del número de participantes, la pertinencia del número, la naturaleza y actitud de los participantes y la subjetividad del mane- 
jo, interpretación y estructuración de la información. Sin embargo, para este caso la asistencia a los talleres fue "relativamente" concurrida, respecto al número total de productores y sobre todo existió la representatividad de cada una de las partes involucradas; por lo que los resultados que se presentan muestran una realidad muy cercana a lo que acontece en esta actividad en la región de estudio. Lo del número de talleres se ha argumentado con antelación.

El primer taller ZOPP, tuvo lugar en las instalaciones de la Universidad Politécnica de Tulancingo, el día 10 de abril de 2013, asistiendo 30 personas. El moderador detalló a los asistentes la finalidad de estos talleres, enfatizando la necesidad de realizar participativamente los cinco instrumentos considerados por la Planeación Participativa y argumentó la importancia que tenía la asistencia y participación continua de los diferentes representantes en cada una de las sesiones a realizarse. El moderador solicitó a los diferentes participantes que escribieran, en tarjetas de trabajo, problemas que enfrenta la producción de forrajes y leche en la región Tulancingo, esto con el fin de elaborar el Árbol de Problemas. Enfatizó que se escribieran, de manera concreta y visible, un problema en cada tarjeta. Después de realizar el ejercicio, dichas tarjetas fueron entregadas al facilitador, y este se encargó de ponerlas en una pizarra, respetando la relación causa-efecto considerando la opinión y validación de los participantes.

Se continuó elaborando el Árbol de Problemas los días 12 y 13 de abril de 2013, estando presentes 32 y 33 personas respectivamente. Se determinó como problemática central: "inexistencia de eslabonamiento productivo del sector lácteo” (Figura 1), con tres problemas comunes y dos específicos para cada uno de los eslabones. Que, en conjunto, ha agudizado los problemas generales de desempleo regional, inseguridad, emigración, pobreza, y debilidad institucional.

Después de ser validado el Árbol de Problemas, se inició con la elaboración del Árbol de Objetivos (Figura 2), que consistió en transformar los problemas a soluciones, en cambiar una situación no deseada a una deseada. Este árbol contiene los ejes estratégicos, líneas de acción y proyectos propuestos por ellos mismo, para la construcción de la MPP (Cuadro 1 y 2), que constituye el elemento central del proceso de planeación, basado en la participación. El Árbol de Objetivos fue concluido y validado en la cuarta sesión del ZOPP, realizada el 24 de abril de 2013 donde asistieron 30 personas.

Cabe destacar la importancia que tuvo la validación en los procesos participativos, de estos dos primeros instrumentos (Árbol de Problemas y Objetivos), al contener la sistematización y estructuración de la información vertida en los talleres por los diferentes participantes, tratando de evitar cualquier tipo de subjetividad en la interpretación y organización de la información. La validación de estos instrumentos fue secuencial y no se podía proseguir con los demás, si primeramente no se autorizaba el anterior.

Este proceso fue importante, porque los participantes se sintieron parte del problema y de la solución al validar, reconociendo su papel y la responsabilidad de cada uno de ellos, pero con la satisfacción de haber sido escuchados y reconocidos por quienes comparten la misma realidad. Particularmente, considero que la dinámica de los talleres fue un resultado importante por sí mismo y medular para una participación más activa y es lo que diferencia la planeación tradicional de la participativa, en el sentido de pertenencia, al ser construidos de abajo hacia arriba. En este sentido las limitantes de la naturaleza y actitud de los participantes, así como subjetividad de la información fueron permeadas de algún modo en los diferentes talleres.

Finalmente, los quinto y sexto talleres ZOPP, se realizaron el 26 y 30 de abril de 2013 con una asistencia de 34 y 35 personas respectivamente, en donde se construyeron y validaron la MPP, el Análisis de Involucrados y la Planeación Operativa de Proyecto. Estas dos últimas no se ilustran en el presente documento, sin embargo si formaron parte del análisis para definir las estrategias para el desarrollo integral de la producción forraje-leche en la región de Tulancingo.

Una vez que se reconocieron las funciones, intereses, fortalezas y debilidades de los participantes, se diseñaron cada uno de los ejes estratégicos o programas específicos con sus respectivas líneas de acción y, éstas, con los proyectos diseñados para resolver una problemática particular por medio de la realización de actividades específicas, como se muestran, a manera de ejemplo, en el Cuadro 1 para el segundo eje estratégico de forraje: recuperación del margen de utilidad; y el Cuadro 2 del quinto eje estratégico para el eslabón de producción de leche: focalización de los programas gubernamentales. Los demás ejes estratégicos, líneas de acción y proyectos que constituyeron la MPP, para ambos eslabones (forraje-leche), se enuncian completos para el primer eslabón de producción de forraje. Sin embargo, para el siguiente eslabón (leche), únicamente se desglosan para los dos ejes estratégicos (provisión de infraestructura adecuada, y fomento a la capacitación de calidad) particulares y los otros tres ejes estratégicos (aumento en la calidad y rendimiento del producto, recuperación del margen de 
utilidad, y focalización de los programas gubernamentales) únicamente son señaladas sus líneas de acción, por la similitud con el encadenamiento productivo de forraje.

\section{a. Producción de forrajes}

Eje estratégico 1. Aumento del rendimiento y calidad de forraje

1.1. Sistema de producción primordialmente de riego (implementación de sistemas de producción alternativos en uso de agua, y adecuación a las condiciones climáticas).

1.2. Capacitación eficiente (aumento en la disponibilidad de tiempo e interés de los productores, y capacitación pública de calidad).

1.3. Tecnificación adecuada (provisión de recursos financieros accesibles, y fomento al desarrollo de tecnología propia).

1.4. Adecuado manejo pos cosecha de forraje (provisión de maquinaria -ensiladoras, molinos, etc.-, y capacitación adecuada en el manejo pos cosecha de forraje).

1.5. Mayor disponibilidad de agua (implementación de sistemas eficientes de captación de aguas fluviales, explotación adecuada de los mantos acuíferos, y desarrollo programas de tratamiento de aguas residuales).

1.6. Uso adecuado y mejora en la calidad de insumos (disminución de los costos de insumos -semillas certificadas, agroquímicos, etc.-, y fomento de estudios de suelo que determinen la potencialidad productiva del lugar).

El eje estratégico 2. Recuperación del margen de utilidad

Recuperación del margen de utilidad de forraje en la región Tulancingo, se ilustra en el Cuadro 1.

Eje estratégico 3. Fomento del trabajo colaborativo

3.1. Impulso del trabajo colectivo (fomento de la cultura de trabajo en equipo, y desarrollo de mecanismos de organización de tiempos).

3.2. Reconocimiento de compromisos de los productores (desarrollo de mecanismos de organización de tiempos, e involucramiento de empresarios en la solución de sus problemas).

3.3. Fomento de la comunicación (reconocimiento de intereses de grupo sobre los particulares -foros de acuerdos-, creación de una organización regional que unifique los intereses del sector, e impulso a la cohesión entre productores).

3.4. Formación de líderes honestos (promoción de la cultura de servicio colectivo, y distribución de los costos de gestión).

Eje estratégico 4. Provisión de infraestructura carretera

4.1. Habilitación de caminos de acceso a las comunidades (conclusión de caminos de acceso a comunidades, y planeación en el diseño de carreteras).

4.2. Mantenimiento oportuno en caminos de terracería (mantenimiento continuo en carreteras de terracería, y provisión de maquinaria).

El eje estratégico 5. Focalización de los programas gubernamentales

Este eje es similar en sus proyectos al que se ilustra en el Cuadro 2, por lo que únicamente se enuncian las líneas de acción.

5.1. Provisión de apoyos financieros dirigidos a la productividad

5.2. Evaluación continua del alcance de los programas como Procampo, Alianza para el campo, etc.

5.3. Adecuación de Trámites y requisitos en tiempo y forma

5.4. Profesionalismo de los programas

\section{b. Producción de leche}

Eje estratégico 1. Aumento en la calidad de la leche

1.1. Identificación de animales apropiados genéticamente para la región

1.2. Diseño de un programa de control de enfermedades

GESTIÓN Y ANÁLISIS DE POLÍTICAS PÚBLICAS, Nueva Época, no 13 enero-junio 2015 - ISSN: 1989-8991 - DOI: 10.24965/gapp.v0i13.10233 
1.3. Acceso a forraje barato y de calidad

1.4. Fomento a la implementación de buenas prácticas de producción

1.5. Conocimiento adecuado de dietas balanceadas

1.6. Aplicación de la normatividad de COPRISEH

Eje estratégico 2. Recuperación del margen de utilidad

2.1. Mejora en el precio de la leche

2.2. Reducción de intermediarios en el acopio de leche -boteros, centros de acopio, etc.- 2.3. Disminución en el costo de insumos

\section{Eje estratégico 3. Provisión de infraestructura adecuada}

3.1. Estímulo a la cultura de buenas prácticas de manejo de producción (reconocimiento de una visión de conjunto -cadena productiva-, y capacitación de productores en buenas prácticas de manejo de la producción).

3.2. Acceso a recursos financieros (aumento de la rentabilidad del sector, disminución del costo de maquinaria de importación por compras de mayoreo, disponibilidad de créditos privados accesibles, y disponibilidad de planes de financiamiento público accesibles).

3.3. Establecimiento de corrales apropiados (planeación de la crianza de animales y producción de leche, y aplicación de la normatividad zoosanitaria).

Eje estratégico 4. Fomento a la capacitación de calidad

4.1. Fomento del interés del empresario (promoción de la cultura empresarial, y alfabetización direccionada a productores de leche).

4.2. Aumento de la disponibilidad de tiempo del productor (adecuación de cursos a horarios disponibles de productores de leche, y desarrollo de mecanismos de organización de tiempos).

4.3. Establecimiento de centros de capacitación local adecuados (capacitación en buenas prácticas de higiene, capacitación en buenas prácticas de manejo de producción de leche, y Formación de recursos humanos calificados en la región).

Eje estratégico 5. Focalización de los programas gubernamentales

Focalización de los programas gubernamentales para la producción de leche-bovino se muestra esquematizado en el Cuadro 2.

La opinión de los diferentes involucrados en los talleres de Planeación Participativa fue importante para la determinación de cada uno de los ejes estratégico, líneas de acción y proyectos, señalados con antelación. Puesto que ello permitió la priorización de necesidades y acciones a seguir para fomentar la producción de forraje y leche y, con ello, lograr mejores condiciones de bienestar de la población objeto de estudio.

Sin embargo, otro hecho importante de resaltar por la implementación de la Planeación Participativa, fue el reconocimiento de los productores de forraje y leche como los principales gestores de su desarrollo. Sin demeritar la responsabilidad que le compete a las diferentes dependencias públicas, así como el apoyo de instituciones educativas, organizaciones gremiales, ciudadanas, entre otras.

En este sentido, las estrategias, líneas y proyectos planteados en este documento, para el sector forraje-leche de la región Tulancingo, responden a una problemática real y actual, que demanda una colaboración coordinada de los diferentes involucrados, pero sobre todo de la voluntad de querer hace las cosas bajo un esquema no tradicional, en donde el compromiso de la ejecución de los proyectos son compartidos por todos aquellos que forman parte tanto del problema como de la solución. Esto implica estrechar relaciones de colaboración entre los sectores productivos, la sociedad, el gobierno e instituciones de docencia e investigación a favor de un desarrollo integral de la producción forraje-leche en la región Tulancingo.

\section{CONCLUSIONES}

Esta investigación se realizó en un momento de incertidumbre institucional que enfrenta el país, donde la población ya no cree en las promesas incumplidas de los servidores públicos. Esta situación repercute en los procesos de planeación donde intervienen dependencias gubernamentales encargadas de fomentar el desarrollo sectorial 
integral, sin lograrlo. Causando a los beneficiarios malestar y falta de interés de participar en los sistemas tradicionales de planeación de arriba hacia abajo.

En este caso la intermediación de la Universidad Politécnica de Tulancingo y la Universidad Autónoma del Estado de Hidalgo, reconocidas por su prestigio académico y de investigación, en la región de estudio, permitió realizar con éxito los talleres ZOPP.

La implementación de la Planeación Participativa, fue una herramienta importante para la identificación de la problemática del eslabonamiento forraje-leche en la región Tulancingo, además del reconocimiento y la generación de sinergias entre los agentes de los diferentes eslabones de la cadena productiva del sector lácteo, la cual existe productiva más no funcional, ni organizacionalmente.

Los diagnósticos de abajo hacia arriba con visión territorial ayudan a identificar potencialidades sectoriales desaprovechadas, como el caso de la actividad láctea en la región Tulancingo, a favor de la diversificación económica, la derrama sectorial y la dinámica regional integral.

El reconocimiento de necesidades comunes, basado en sistemas participativos, como parte de una cadena productiva delimitada territorialmente, fue la base para diseñar estrategias de desarrollo asertivas e integrales de corto y largo plazo a favor de un desarrollo regional armónico y sustentable.

Al existir el involucramiento de los diferentes actores se generaron sinergias positivas que no sólo propician el crecimiento económico de esta actividad productiva, sino también un mayor bienestar entre la población que consolida el tejido social, necesario para el fortalecimiento de un sistema institucional local, actualmente deteriorado.

Finalmente, las dependencias públicas reconocieron el papel central que tienen como instituciones impulsoras del desarrollo regional integral, coordinado horizontal y verticalmente y la importancia de diseñar e implementar políticas públicas regionales innovadoras, consensuadas, a favor de la productividad sectorial. De igual manera, las instituciones académicas reconocieron su papel como mediador en los procesos participativos y la capacidad de potencializar el desarrollo, como formadoras de capital humano y generadoras de conocimiento.

FIGURA 1. ÁRBOL DE PROBLEMAS FORRAJE-LECHE

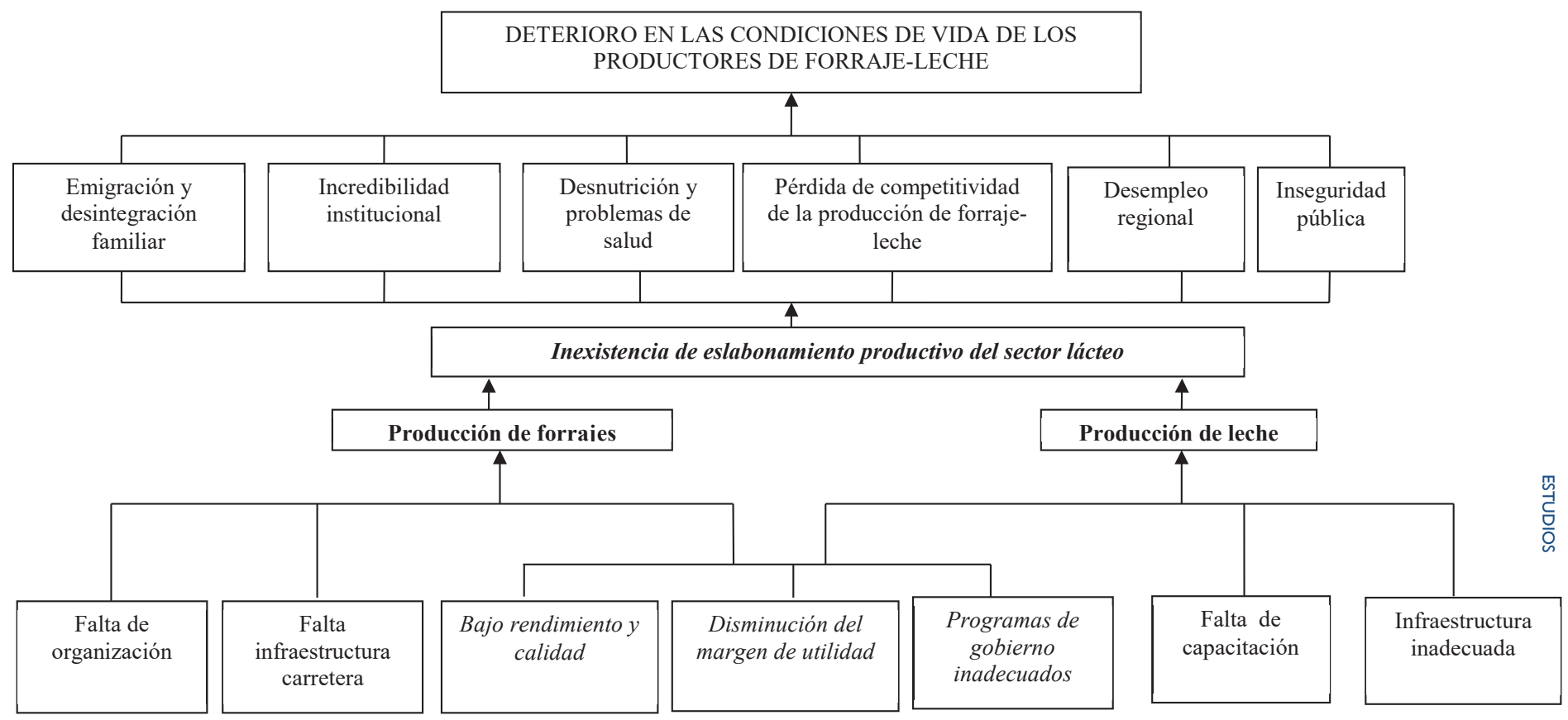

Fuente: Elaboración propia con información generada en los talles ZOPP. 
FIGURA 2. ÁRBOL DE OBJETIVOS FORRAJE-LECHE

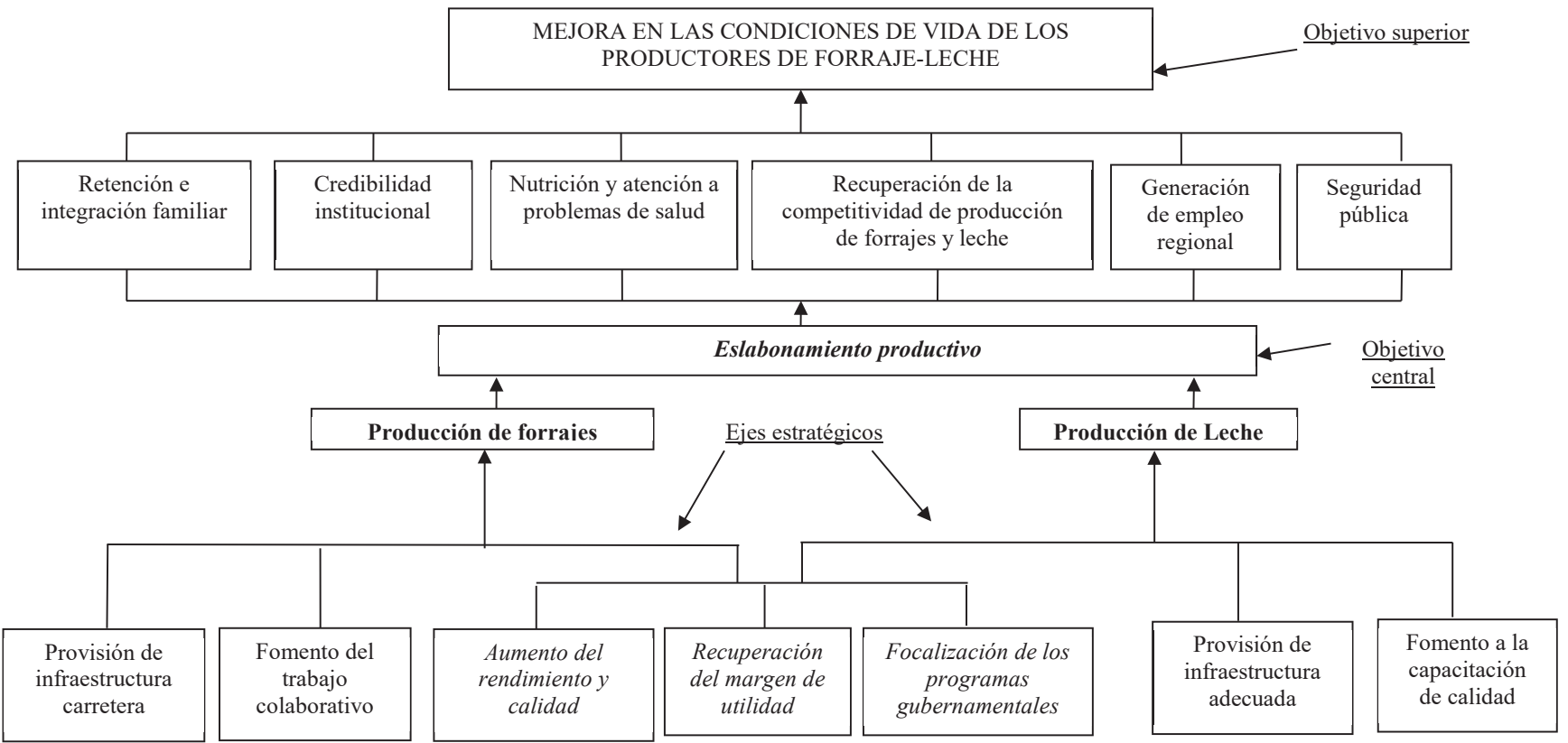

Fuente: Elaboración propia con información generada en los talles ZOPP.

CUADRO 1. MATRIZ DE PLANEACIÓN DE PROYECTO (MPP): FORRAJE-REGIÓN TULANCINGO

\begin{tabular}{|c|c|c|c|}
\hline Estrategia del Proyecto & $\begin{array}{l}\text { Indicadores Verificables Objeti- } \\
\text { vamente }\end{array}$ & Fuentes de Verificación & Supuestos \\
\hline \multicolumn{4}{|l|}{$\begin{array}{l}\text { Objetivo superior: Mejora en las con- } \\
\text { diciones de vida de la cadena produc- } \\
\text { tiva del sector lácteo } \\
\text { (forraje-leche-queso) }\end{array}$} \\
\hline \multicolumn{4}{|l|}{$\begin{array}{l}\text { Nombre del programa: } \\
\text { 2. Recuperación del margen de utili- } \\
\text { dad }\end{array}$} \\
\hline $\begin{array}{l}\text { Objetivo del programa: } \\
\text { 2. Recuperar el margen de utilidad de } \\
\text { la actividad forrajera regional }\end{array}$ & $\begin{array}{l}\text { Aumento del margen de utilidad } \\
\text { de la actividad forrajera regional }\end{array}$ & $\begin{array}{l}\text { Estudios de estimación } \\
\text { de margen de utilidad de } \\
\text { forraje por diferentes ins- } \\
\text { tituciones }\end{array}$ & $\begin{array}{l}\text { Existe participación institucional } \\
\text { integral }\end{array}$ \\
\hline \multicolumn{4}{|l|}{$\begin{array}{l}\text { Líneas de acción: } \\
\text { 2.1. Mejora en el precio del forraje } \\
\text { 2.2. Disminución del costo de insumos } \\
\text { 2.3. Reducción de intermediarios en } \\
\text { proveeduría de insumos }\end{array}$} \\
\hline $\begin{array}{l}\text { Proyectos: } \\
\text { 2.1.1. Concertación de precios entre } \\
\text { eslabones de la cadena productiva }\end{array}$ & $\begin{array}{l}\text { Mayor precio de venta del forra- } \\
\text { je en la región }\end{array}$ & $\begin{array}{l}\text { Estadísticas de la Sagar- } \\
\text { pa y presidencias muni- } \\
\text { cipales }\end{array}$ & Existe voluntad de colaboración \\
\hline 2.1.2. Estímulo a la competencia leal & $\begin{array}{l}\text { Estabilidad del precio de venta de } \\
\text { forraje }\end{array}$ & $\begin{array}{l}\text { Estadísticas de la Sagarpa } \\
\text { y presidencias municipa- } \\
\text { les }\end{array}$ & $\begin{array}{l}\text { Hay formación de valores y vo- } \\
\text { luntad de colaboración }\end{array}$ \\
\hline $\begin{array}{l}\text { 2.2.1. Implementación de convenios } \\
\text { de tarifas de energía eléctrica }\end{array}$ & $\begin{array}{l}\text { Número de convenios entre pro- } \\
\text { ductores de forraje y CFE sobre } \\
\text { tarifas de energía }\end{array}$ & $\begin{array}{l}\text { Estadísticas de la Sagar- } \\
\text { pa. CFE y presidencias } \\
\text { municipales }\end{array}$ & $\begin{array}{l}\text { Existe la voluntad política y or- } \\
\text { ganización para este tipo de } \\
\text { convenios }\end{array}$ \\
\hline $\begin{array}{l}\text { 2.2.2. Concertación de precios de com- } \\
\text { bustibles productivos }\end{array}$ & $\begin{array}{l}\text { Número de apoyos a producto- } \\
\text { res de forraje de combustible } \\
\text { (diesel y gasolina) }\end{array}$ & $\begin{array}{l}\text { Estadísticas de desarrollo } \\
\text { agropecuario municipal }\end{array}$ & $\begin{array}{l}\text { Existe la voluntad política y or- } \\
\text { ganización para este tipo de } \\
\text { convenios }\end{array}$ \\
\hline $\begin{array}{l}\text { 2.2.3. Desaparición de monopolios de } \\
\text { insumos (semillas certificadas, agro- } \\
\text { químicos) }\end{array}$ & $\begin{array}{l}\text { Número de proveedores de insu- } \\
\text { mos en la región }\end{array}$ & $\begin{array}{l}\text { Estadísticas de desarrollo } \\
\text { económico o comercio a } \\
\text { nivel municipal }\end{array}$ & $\begin{array}{l}\text { Existe voluntad política y partici- } \\
\text { pación institucional integral }\end{array}$ \\
\hline
\end{tabular}




\begin{tabular}{|c|c|c|c|}
\hline $\begin{array}{l}\text { 2.3.1. Organización para compras al } \\
\text { mayoreo }\end{array}$ & $\begin{array}{l}\text { Número de organizaciones forra- } \\
\text { jeras en la región }\end{array}$ & $\begin{array}{l}\text { Estadísticas de la direc- } \\
\text { ción de desarrollo agro- } \\
\text { pecuario municipal }\end{array}$ & $\begin{array}{l}\text { Existe la capacidad de organiza- } \\
\text { ción entre los productores de } \\
\text { forraje }\end{array}$ \\
\hline $\begin{array}{l}\text { 2.3.2. Acercamiento de proveeduría } \\
\text { de insumos a comunidades forrajeras }\end{array}$ & $\begin{array}{l}\text { Número de proveedores de insu- } \\
\text { mos en las localidades forrajeras } \\
\text { de la región }\end{array}$ & $\begin{array}{l}\text { Estadísticas de desarrollo } \\
\text { económico o comercio a } \\
\text { nivel municipal }\end{array}$ & $\begin{array}{l}\text { Hay potencialidad de mercado } \\
\text { en las localidades productoras } \\
\text { de forraje }\end{array}$ \\
\hline \multicolumn{4}{|l|}{ Actividades } \\
\hline \multicolumn{4}{|c|}{ 2.1.1.1. Concertar precios entre eslabones de la cadena productiva } \\
\hline \multicolumn{4}{|l|}{ 2.1.2. 1. Estimular la competencia leal } \\
\hline \multicolumn{4}{|c|}{ 2.2.1.1. Implementar convenios de tarifas de energía eléctrica } \\
\hline \multicolumn{4}{|c|}{ 2.2.2.1. Concertar precios de combustibles productivos } \\
\hline \multicolumn{4}{|c|}{ 2.2.3.1. Desaparecer monopolios de insumos (semillas certificadas, agroquímicos) } \\
\hline \multicolumn{4}{|l|}{ 2.3.1. 1. Organizar para compras al mayoreo } \\
\hline \multicolumn{4}{|c|}{ 2.3.2. Acercar la proveeduría de insumos a comunidades forrajeras } \\
\hline
\end{tabular}

Fuente: Elaboración propia con información generada en los talles ZOPP.

aSagarpa: Secretaría de Agricultura, Ganadería, Desarrollo Rural, Pesca y Alimentación

${ }^{\mathrm{b}} \mathrm{CFE}$ : Comisión Federal de Electricidad.

CUADRO 2. MATRIZ DE PLANEACIÓN DE PROYECTO (MPP): LECHE-REGIÓN TULANCINGO

\begin{tabular}{|c|c|c|c|}
\hline Estrategia del Proyecto & $\begin{array}{c}\text { Indicadores Verificables } \\
\text { Objetivamente }\end{array}$ & Fuentes de Verificación & Supuestos \\
\hline $\begin{array}{l}\text { Objetivo superior: Mejora en las } \\
\text { condiciones de vida de la cadena } \\
\text { productiva del sector lácteo } \\
\text { (forraje-leche-queso) }\end{array}$ & & & \\
\hline $\begin{array}{l}\text { Nombre del programa: } \\
\text { 5. Focalización de los programas } \\
\text { gubernamentales }\end{array}$ & & & \\
\hline $\begin{array}{l}\text { Objetivo del programa: } \\
5 . \text { Focalizar los programas guber- } \\
\text { namentales }\end{array}$ & $\begin{array}{l}\text { Mayor cobertura e impacto } \\
\text { de los programas guberna- } \\
\text { mentales }\end{array}$ & $\begin{array}{l}\text { Estudio de disponibilidad y efectivi- } \\
\text { dad de los programas públicos }\end{array}$ & $\begin{array}{l}\text { Existe compromiso del gobier- } \\
\text { no y participación institucional } \\
\text { integral }\end{array}$ \\
\hline $\begin{array}{l}\text { Líneas de acción: } \\
\text { 5.1. Provisión de apoyos financie- } \\
\text { ros dirigidos a la productividad } \\
\text { m.2. Protección normativa del } \\
\text { mercado } \\
\text { 5.3. Adecuación de Trámites y re- } \\
\text { quisitos en tiempo y forma } \\
\text { 5.4. Profesionalismo de los pro- } \\
\text { gramas } \\
\text { 5.5. Estimulación del sector }\end{array}$ & & & \\
\hline $\begin{array}{l}\text { Proyectos } \\
\text { 5.1.1. Disponibilidad de crédito } \\
\text { para modernización de infraes- } \\
\text { tructura (ordeñadoras, corrales, } \\
\text { etc.) }\end{array}$ & $\begin{array}{l}\text { Aumento en disponibilidad } \\
\text { de financiamiento público } \\
\text { para adquisición de infraes- } \\
\text { tructura de las plantas que- } \\
\text { seras }\end{array}$ & $\begin{array}{l}\text { Estadísticas de recursos financieros } \\
\text { para infraestructura en la región pro- } \\
\text { porcionadas por Sedecoc, etc. }\end{array}$ & $\begin{array}{l}\text { Existe la voluntad y el compro- } \\
\text { miso por instituciones financie- } \\
\text { ras de desarrollo }\end{array}$ \\
\hline $\begin{array}{l}\text { 5.1.2. Determinación del requeri- } \\
\text { miento financiero del sector }\end{array}$ & $\begin{array}{l}\text { Aumento en disponibilidad } \\
\text { de financiamiento público } \\
\text { dirigido a la productividad }\end{array}$ & $\begin{array}{l}\text { Estadísticas sobre disponibilidad de } \\
\text { recursos de instituciones financieras } \\
\text { de desarrollo } \\
\text { dirigidas al sector quesero regional }\end{array}$ & $\begin{array}{l}\text { Existe el interés institucional por } \\
\text { determinar los requerimientos } \\
\text { financieros del sector quesero } \\
\text { regional }\end{array}$ \\
\hline $\begin{array}{l}\text { 5.2.1 Impulso de leyes que eviten } \\
\text { la entrada de insumos producti- } \\
\text { vos de dudosa procedencia }\end{array}$ & $\begin{array}{l}\text { Disminución de la entrada } \\
\text { de insumos de dudosa pro- } \\
\text { cedencia }\end{array}$ & $\begin{array}{l}\text { Estadísticas de importaciones de } \\
\text { insumos vinculados al sector por } \\
\text { Bancomext } \mathrm{d}^{\mathrm{d}} \text { la SE }\end{array}$ & $\begin{array}{l}\text { Existe la voluntad política para } \\
\text { aplicación la ley }\end{array}$ \\
\hline $\begin{array}{l}\text { 5.2.2. Aplicación de la normativi- } \\
\text { dad del contenido nutrimental en } \\
\text { el etiquetado del producto final }\end{array}$ & $\begin{array}{l}\text { Disponibilidad nutrimental } \\
\text { en el etiquetado del pro- } \\
\text { ducto final }\end{array}$ & $\begin{array}{l}\text { Estadística de la Sedeco e informes } \\
\text { municipales }\end{array}$ & $\begin{array}{l}\text { Existe la decisión de aplicación } \\
\text { de la normatividad }\end{array}$ \\
\hline $\begin{array}{l}\text { 5.3.1. Impulso a la coordinación } \\
\text { institucional en los diferentes ni- } \\
\text { veles de gobierno }\end{array}$ & $\begin{array}{l}\text { Cobertura de convocatorias } \\
\text { de programas gubernamen- } \\
\text { tales }\end{array}$ & $\begin{array}{l}\text { Estudios de seguimiento a convocato- } \\
\text { rias públicas en los diferentes niveles } \\
\text { de gobierno }\end{array}$ & $\begin{array}{l}\text { Existe la voluntad y la coordina- } \\
\text { ción interinstitucional, así como } \\
\text { el interés por parte de institucio- } \\
\text { nes de investigación para reali- } \\
\text { zar este tipo de estudios }\end{array}$ \\
\hline
\end{tabular}




\begin{tabular}{|c|c|c|c|}
\hline $\begin{array}{l}\text { 5.3.2. Apoyo para minimizar los } \\
\text { costos de elaboración de proyec- } \\
\text { tos }\end{array}$ & $\begin{array}{l}\text { Aumento en el número de } \\
\text { proyectos concursables en } \\
\text { los programas públicos }\end{array}$ & $\begin{array}{l}\text { Estadísticas de proyectos concursa- } \\
\text { bles y beneficiados proporcionadas } \\
\text { por SE }\end{array}$ & $\begin{array}{l}\text { Existe la voluntad y la coordina- } \\
\text { ción interinstitucional }\end{array}$ \\
\hline $\begin{array}{l}\text { 5.3.3. Adecuación de las reglas de } \\
\text { operación de los programas }\end{array}$ & $\begin{array}{l}\text { Aumento en el número de } \\
\text { productores lecheros bene- } \\
\text { ficiados por programas gu- } \\
\text { bernamentales en la región }\end{array}$ & $\begin{array}{l}\text { Estadísticas de productores benefi- } \\
\text { ciados proporcionadas por desarrollo } \\
\text { económico de presidencias municipa- } \\
\text { les. }\end{array}$ & $\begin{array}{l}\text { Existe la voluntad y la coordina- } \\
\text { ción interinstitucional }\end{array}$ \\
\hline $\begin{array}{l}\text { 5.4.1. Eliminación de intereses po- } \\
\text { líticos en la asignación de recur- } \\
\text { sos (sin color de partido) }\end{array}$ & $\begin{array}{l}\text { Mayor cobertura de los pro- } \\
\text { gramas gubernamentales } \\
\text { independientes a partidos } \\
\text { políticos }\end{array}$ & $\begin{array}{l}\text { Estudio de asignación de los recursos } \\
\text { públicos sectoriales en la región por } \\
\text { partido político }\end{array}$ & $\begin{array}{l}\text { Existe el interés por parte de ins- } \\
\text { tituciones de investigación para } \\
\text { realizar este tipo de estudios }\end{array}$ \\
\hline $\begin{array}{l}\text { 5.4.2. Transparencia de la infor- } \\
\text { mación de los programas }\end{array}$ & $\begin{array}{l}\text { Cobertura de convocatorias } \\
\text { de programas gubernamen- } \\
\text { tales }\end{array}$ & $\begin{array}{l}\text { Estudio comparativo municipal so- } \\
\text { bre oportunidad y trasparencia de } \\
\text { la información de las convocatorias } \\
\text { públicas }\end{array}$ & $\begin{array}{l}\text { Existe el interés por parte de ins- } \\
\text { tituciones de investigación para } \\
\text { realizar este tipo de estudios }\end{array}$ \\
\hline $\begin{array}{l}\text { 5.5.1. Implementación de una po- } \\
\text { lítica fiscal, sanitaria y comercial } \\
\text { estimulante }\end{array}$ & $\begin{array}{l}\text { Aumento en el registro de } \\
\text { hatos lecheros }\end{array}$ & $\begin{array}{l}\text { Estadísticas del SAT }{ }^{f}, \mathrm{SSA}^{g}, \mathrm{SE} \text {, Presi- } \\
\text { dencias municipales, etc. }\end{array}$ & $\begin{array}{l}\text { Existe la voluntad para imple- } \\
\text { mentar acuerdos entre produc- } \\
\text { tores de leche y dependencias } \\
\text { gubernamentales }\end{array}$ \\
\hline $\begin{array}{l}\text { 5.5.2. Aplicación de la normativi- } \\
\text { dad para todos }\end{array}$ & $\begin{array}{l}\text { Mayor otorgamiento de } \\
\text { permisos y licencias }\end{array}$ & $\begin{array}{l}\text { Estadísticas del SAT, SSA, SE, Presi- } \\
\text { dencias municipales, etc. }\end{array}$ & $\begin{array}{l}\text { Existe el interés por regularizar } \\
\text { el sector lechero }\end{array}$ \\
\hline $\begin{array}{l}\text { 5.5.3. Creación de programas } \\
\text { gubernamentales incluyentes de } \\
\text { promoción de mercados }\end{array}$ & $\begin{array}{l}\text { Mayor proyección y nivel de } \\
\text { ventas de leche en la región } \\
\text { y el mercado nacional }\end{array}$ & $\begin{array}{l}\text { Estadísticas de ventas y eventos de la } \\
\text { Sedeco }\end{array}$ & $\begin{array}{l}\text { Existe la disposición empresarial } \\
\text { y coordinación interinstitucional }\end{array}$ \\
\hline \multicolumn{4}{|l|}{ Actividades } \\
\hline \multicolumn{4}{|c|}{ 5.1.1.1. Disponer de crédito para modernización de infraestructura (ordeñadoras, corrales, etc.) } \\
\hline \multicolumn{4}{|c|}{ 5.1.2.1. Determinar el requerimiento financiero del sector } \\
\hline \multicolumn{4}{|c|}{ 5.2.1.1. Impulsar leyes que eviten la entrada de insumos productivos de dudosa procedencia } \\
\hline \multicolumn{4}{|c|}{ 5.2.2.1. Aplicar la normatividad del contenido nutrimental en el etiquetado } \\
\hline \multicolumn{4}{|c|}{ 5.3.1.1. Impulsar la coordinación institucional en los diferentes niveles de gobierno } \\
\hline \multicolumn{4}{|c|}{ 5.3.2.1. Apoyar los costos de elaboración de proyectos } \\
\hline \multicolumn{4}{|c|}{ 5.3.3.1. Adecuar las reglas de operación de los programas } \\
\hline \multicolumn{4}{|c|}{ 5.4.1.1. Eliminar intereses políticos en la asignación de recursos (sin color de partido) } \\
\hline \multicolumn{4}{|c|}{ 5.4.2.1. Transparentar la información de los programas } \\
\hline \multicolumn{4}{|c|}{ 5.5.1.1. Implementar una política fiscal, sanitaria y comercial estimulante } \\
\hline \multicolumn{4}{|c|}{ 5.5.2.1. Aplicar la normatividad para todos } \\
\hline \multicolumn{4}{|c|}{ 5.5.3. 1. Crear programas gubernamentales incluyentes de promoción } \\
\hline
\end{tabular}

Fuente: Elaboración propia con información generada en los talles ZOPP.

c Sedeco: Secretaría de Desarrollo Económico-Hidalgo

${ }^{\mathrm{d}}$ Bancomext: Banco de Comercio Exterior.

e SE: Secretaría de Economía

fSAT: Servicio de Administración Tributaria

gSSA: Secretaría de Salubridad y Asistencia

\section{BIBLIOGRAFÍA}

Banco Mundial (2005), México 2006-2012. Creando las bases para un crecimiento equitativo. En línea <http:// www.bancomundial.org> (consulta 10 de marzo de 2014).

Blakely, E. y Green, L. N. (2010), Planning local economic development: theory and practice, Thousand Oaks: SAGE Publications Inc. En línea: http://dx.doi.org/10.1016/s0264-2751(96)90039-6 (consulta: 25 de abril de 2014).

Organización de la Naciones Unidas para la Agricultura y la Alimentación (FAO) (2013), División de Infraestructuras rurales y agroindustriales: Capacitación sobre la cadena de valor. En línea: http://www.fao.org/ag/ags/desarrollo-agroempresarial/capacitacion-sobre-la-cadena-de-valor/es/?no_cache=1 (consulta: 13 de febrero de 2013).

Forster, R. y Osterhaus, J. (1995), Marco orientativo para la ejecución de proyectos, México, Cooperación Técnica Alemana, GTZ.

Geilfus, F. (2000), 80 herramientas para el desarrollo participativo. México: IICA. 
Giménez, G. (1994), “Comunidades primordiales y modernización en México”, en Giménez, G. y Pozas, R. (coords.), Modernización e identidades sociales, México: UNAM, pp. 152-166.

GTZ (1995). Marco orientativo para la ejecución de proyectos de la Cooperación Técnica alemana a través de la GTZ, México, Agencia Alemana de Cooperación Técnica: Team Technologies (GTZ).

GTZ., O. J. y Salzer, O. W. (1995), Diferenciación según género en el ciclo del proyecto. Indicaciones para la planificación, monitoreo y evaluación.

Instituto Nacional de Estadística y Geografía (Inegi)

- (2000). Marco geoestadístico: Conjunto de datos vectoriales de la carta topográfica, 1:50 000, México.

- (2010). Censo de Población y Vivienda. México, En línea: http://www.censo2010.org.mx/ (consulta: 13 de abril de 2013).

Leurs, R. (1996), “Current challenges facing participatory rural appraisal, Public administration and development”, 16: 57-72. En línea: http://dx.doi.org/10.1002/(sici)109962x(199602)16:1<57::aidad853>3.3.c0;2q (consulta: 10 de julio de 2014).

Martínez C. P., Colino, S. F., y Gómez, M. Á. (2014), "Pobreza y políticas de desarrollo rural en México”, Estudios Sociales, XXII (43):11-35.

Morales, F. (1998), Desarrollo local: principios, metodologías y experiencias, México, Fundación Friedich.

Olvera, R. A. (1999), “Los modos de la recuperación contemporánea de la línea de sociedad civil”, en Alberto Olvera (coord.), La sociedad civil. De la teoría a la realidad, México: El Colegio de México, pp. 27-53.

Organización para la Cooperación Económica y el Desarrollo (OCDE) (2011), "Políticas de desarrollo regional: coordinación entre diferentes niveles de gobierno" en Mejores políticas para el desarrollo: Perspectivas OCDE sobre Chile, OCDE Published, Chile: 155-170. En línea: http://dx.doi.org/10.1787/9789264095755-10-es (consulta: 11 de enero de 2014).

Osorio, M. E. (2009), “La participación ciudadana en asociaciones civiles y la democratización de la sociedad: dos casos oaxaqueños”, en Chávez C., J. C. y Castro, G. M. (coords.): Cultura de participación y construcción de ciudadanía, México: Miguel Ángel Porrúa. Pp. 167-185.

Pardo, M. C. (1994), “La gestión municipal, ¿motor o freno para el ejercicio democrático?”, en Merino, M. (coords.): En busca de la democracia municipal. La participación ciudadana en el gobierno local mexicano. México: El Colegio de México, Pp. 253-282.

Ramírez, G. y Guillermo, A. (2004), Guía metodológica para la formulación y evaluación de proyectos de educación ambiental bajo un enfoque participativo, México: Chapingo (Centro Regional Universitario del Noreste).

Rodríguez, E., Ramírez, G. C. J. y Restrepo, B. L. F. (2015), “Propuesta metodológica para la formulación participativa de programas de desarrollo agropecuario local”, Revista Luna Azul, 40:224-239. En línea: http://dx.doi. org/10.17151/luaz.2015.40.15 (consulta: 21 de marzo de 2015).

Rodríguez, S. R. (1990), Determinación y priorización de problemas a nivel de la comunidad, San Andrés, El Salvador, CENTA.

Secretaría de Agricultura, Ganadería, Desarrollo Rural, Pesca y Alimentación (Sagarpa) (2013). Sistema producto bovino-leche, cadena productiva. En línea: http://spbl.org.mx/bovinosleche_spbl.php (consulta: 23 de mayo de 2013).

Sánchez, B. A., Tovar, G. E. y Sánchez, M. A. (2003), "Evaluación del desempeño de los gobiernos municipales de Jalisco. Hacia una política regional de fortalecimiento municipal” Revista Gestión Municipal, 1(1): 50-67.

Schönhuth, M. (1994), Participatory learning approaches: rapid rural appraisal, participatory appraisal. Rossdorf, Cooperación Técnica Alemana.

Servicio de Información Agroalimentaria y Pesquera (SIAP) (2013), Cierre de la producción agrícola y pecuaria por estados y municipios. En línea: http:/www.siap.gob.mx/index.php?option=com wrapper\&view=wrapper\&Itemid=350 (consulta: 15 de junio de 2014).

Silva, I. (2003). Metodología para la elaboración de estrategias de desarrollo local. Chile, Comisión Económica para América Latina y el Caribe (Cepal). 
Terrones, C. A. (2013). “Planeación participativa para elaborar un plan de desarrollo municipal: el caso de Acaxochitlán, Hidalgo”. Economía, Sociedad y Territorio. Vol. XII, No. 42. Zinacantepec, México. Pp. 521-559.

Terrones, C. A. y Sánchez, T. Y. (2010), Planeación participativa: Teoría y práctica. México: Plaza y Valdés-Universidad Autónoma del Estado de Hidalgo.

Uemura, T. (1999), "Participation, civil society organizations and sustainable development”, Memoirs of Nara University, 28:43-75.

Vázquez, B. A. y Conturruero, R. C. (1994), Notas sobre la estrategia del desarrollo territorial, Cuadernos de Aguilar Núm. 5, textos de apoyo, Universidad Autónoma de Madrid, España.

\section{Agradecimientos}

Estudio realizado con apoyo PROMEP/103-5/1273398 (2012) “Evaluación de proyectos productivos y conformación de redes en la región Tulancingo". 